\title{
Accelerometer-assisted PPG Measurement During Physical Exercise Using the LAVIMO Sensor System
}

\author{
Susana Aguiar Santos, Boudewijn Venema, Steffen Leonhardt \\ Philips Chair for Medical Information Technology, Helmholtz-Institute for Biomedical Engineering, RWTH Aachen \\ University, Pauwelsstrasse 20, 52074 Aachen, Germany
}

Corresponding author: santos@hia.rwth-aachen.de

\begin{abstract}
A method is presented for long-term monitoring of vital signs tested during physical exercise. The system is based on reflective photoplethysmography (PPG), whose main component is a micro-optoelectronic sensor. The sensor is sealed within a biocompatible otoplastic housing so that it can be placed in the external auditory canal. The electronic device has a Bluetooth connection which enables to record/visualize the PPG signal on a personal computer or SmartPhone.

This technology was tested indoors with a subject running on a treadmill at different speeds. The PPG signal was recorded together with an ECG used as a reference, and with an accelerometer to monitor and record motor activity. The accelerometer data were later used to reduce motion artifacts in the PPG signal.

The results show that the system has potential to monitor cardiac activity at moderate speed (up to $4 \mathrm{~km} / \mathrm{h}$ ), but with increasing speed (i.e. running) the motion artifacts dominate the PPG. Therefore, additional studies on signal processing are needed to actively reduce motion artifacts, including the accelerometer data.
\end{abstract}

Keywords: Photoplethysmography (PPG), heart activity, long-term monitoring, health care, sports, physical activity.

\section{Introduction}

In view of the health benefits associated with physical exercise (especially for the cardiovascular system), sports are increasingly popular and are becoming part of the daily routine of patients. Nevertheless, sports are not practiced only for personal healthcare but also for competitions, to achieve physical goals, or even due to pressure from e.g. parents and trainers. In some cases excessive sports activity without appropriate control can be hazardous, leading to overtraining, breakdown and stress [2] or, in the most serious cases, even to sudden death [7]. Therefore, monitoring of vital signs (such as cardiac and respiratory activity) during sports is important.

We present a monitoring system that can be used by patients in their daily routine, including their sports or leisure activities. The system is based on long-term monitoring of vital signs by means of an ear sensor (the 'LAVIMO' system) presented in $[13,14,15]$ which was developed in the projects 'In-Ear 24/7 monitoring system for vital signs' (IN-MONIT) and 'LAVIMO'. The aim is to provide long-term monitoring of vital signs, such as heart rate or respiratory activity. In this paper only cardiac activity is analyzed.

All sport activities and daily tasks imply some movement. Therefore, the monitoring device needs to be robust, mobile, inconspicuous, and of suitable size and weight. In a PPG system, motion artifacts induced by acceleration are a limiting factor. However, the use of an accelerometer to track external disturbances together with advanced signal processing techniques can help to circumvent this problem.

\section{Materials and Methods}

\subsection{PPG fundamentals}

Photoplethysmography (PPG) is a non-invasive optical technique used to investigate subdermal perfusion. Measurements performed at the skin surface detect changes in blood volume in the microvascular bed [1]. PPG is used to monitor vital parameters such as pulse rate, arterial blood oxygen saturation $\left(\mathrm{SpO}_{2}\right)$ and/or respiratory rate.

Most conventional systems work in a transmission mode. Because they detect the number of photons which pass through tissue, these systems can only be applied to the thinner parts of the body (e.g. finger, earlobe). Moreover, this method has some drawbacks: for example, no valid measurement can be made on the finger in case of shock-induced stress (centralization), since the endogenous control of the human body leads to (primary) perfusion of vital organs. Another disadvantage of the conventional systems is that they are not suitable for healthcare applications since daily activities cause considerable motion artifacts. Therefore, we investigated a reflective, multi-wavelength PPG sensor that allows PPG measurement in the outer ear canal (the tragus). Since the ear is very close to the trunk, a valid measurement is expected even in case of centralization. In addition, because 

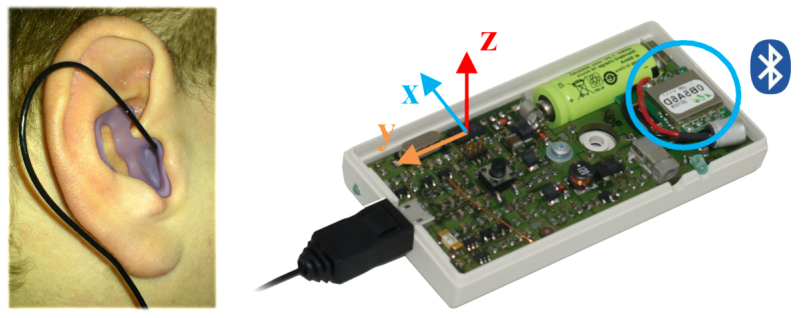

Figure 1: Left: Customized in-ear PPG sensor. Right: Sensor interface device: the LAVIMO Bluetooth system, showing where the Bluetooth and 3-axis accelerometer are placed.

the MedIT in-ear system is very comfortable to wear it can be adapted for various disciplines, including health care and sports.

Our PPG sensor consists of a micro-optoelectronic remission sensor chip, comprised of two light emitting diodes (LED), one red and one near-infrared (wavelengths of $760 \mathrm{~nm}$ and $900 \mathrm{~nm}$, respectively) and a silicon photodiode. The light emitted by these two LEDs is absorbed, transmitted, reflected and scattered by skin, tissue and blood $[3,11]$. The photodiode receives this reflected light.

Since reflectance mode PPG has different signal characteristics compared with transmission mode PPG (i.e. different AC-to-DC ratio), no suitable commercial electronic interface was available. Therefore, we developed a high-performance measurement device, providing 24 bit analog-to-digital converter, sampling rates up to $200 \mathrm{~Hz}$, intelligent LED current control and ambient light compensation (Fig. 1; right).

PPG is based on the fact that the measured light intensity $(I)$ decreases exponentially from the initial light intensity $\left(I_{0}\right)$ with the distance $(x-a)$ passed through the absorbing medium. This is described by Beer-Lambert's Law:

$$
I(x)=I_{0} e^{-\varepsilon(\lambda) c(x-a)}
$$

where $\varepsilon$ is the extinction coefficient, $c$ is the concentration of the absorbing substance, and $\lambda$ is the wavelength. At every cardiac systole, the pulse waves propagating through the arteries lead to shifts in local blood volume. The magnitude of the PPG signal depends on the blood volume in the capillary, which increases during systole and decreases during diastole. Poorly perfused tissue scatters more photons back than does well-perfused tissue. Although PPG signals are usually measured at peripheral areas (such as fingertips, toes and earlobes) where the pulses can be easily detected, measurement on the forehead is also possible [1].

The PPG waveform is composed of 'AC' and ' $\mathrm{DC}$ ' components. The 'AC' pulsatile waveform is attributed to synchronous cardiac changes in blood volume with each heart beat [1], while the 'DC' is the
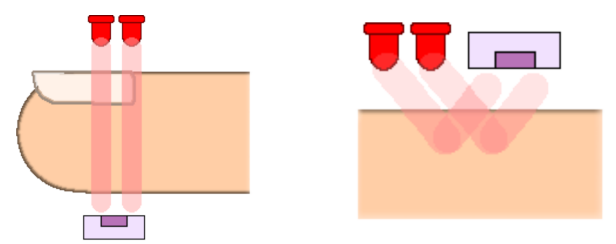

Figure 2: Types of PPG sensors. Left: the transmission mode (used, e.g., in a pulse oximeter); Right the reflectance mode (used in our in-ear PPG sensor).

static component of the signal, also containing slow frequencies due to respiration, sympathetic nervous system activity and thermoregulation [3, 9]. Although some components of a PPG signal are still not fully understood, it is generally accepted that the signals provide important information on the cardiovascular system [5].

\section{$2.2 \quad$ In-ear PPG measuring strategy}

Due to the different physiological characteristics of finger and ear, commercial transmitting sensors cannot be used for in-ear application. Therefore, together with our partner from CiS Erfurt GmbH, we developed a micro-optoelectronic remission sensor chip, which is sealed into an otoplastic housing. Whereas classic systems (e.g. pulse oximeters) use a transmission mode sensor, our system works in a reflectance mode (Fig. 2).

The anatomy of the ear differs from person to person. To decrease motion artifacts the in-ear sensor must fit into the ear as closely as possible. Therefore, for this project, a customized PPG sensor was made to perfectly fit the person involved in the experiment (Fig. 1; left).

\subsection{Data acquisition technology}

The long-term monitoring system used in the present study is the LAVIMO Bluetooth version (Fig. 1; right). The Bluetooth chip (Parani ${ }^{\text {TM }}$ ESD200) provides a Bluetooth connection within a range of 30 meters, which allows easy connection to a personal computer for, e.g. indoor sports, etc. For an outdoor environment, the system can be connected, for instance, to a SmartPhone for signal processing and long-term recording.

The LAVIMO system also incorporates a 3-axis accelerometer (LIS3LV02DL, STMicroelectronics) based on a micro-electromechanical system. The accelerometer data can be used in the signal processing of the PPG signal, with the aim to detect and reduce movement artifacts. 

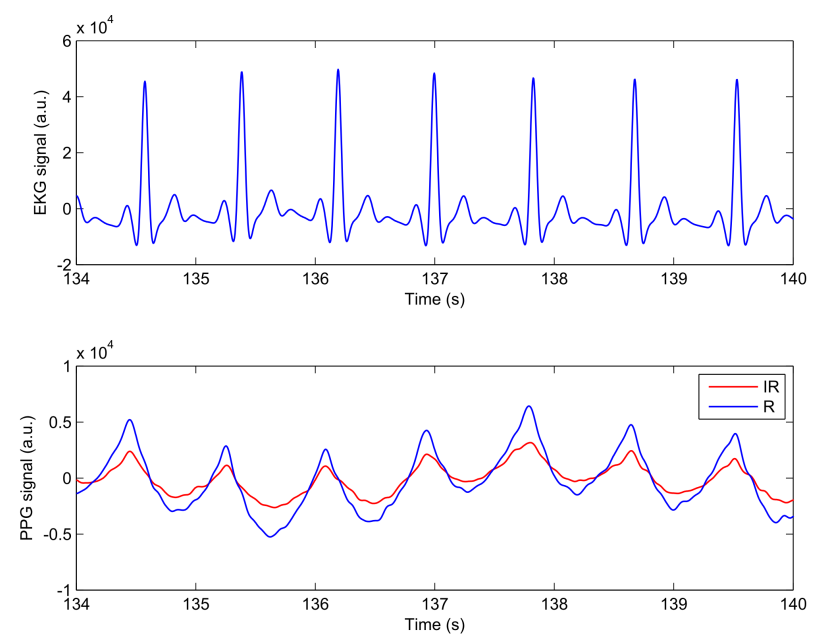

Figure 3: Obtained ECG and PPG signals of a healthy subject at $0 \mathrm{~km} / \mathrm{h}$.

\subsection{Strategies for reduction of movement artifacts}

The corruption of a PPG signal through light or movement of the subject is a limiting factor in a PPG system. These artifacts mask the PPG signal, potentially leading to faulty interpretation of the PPG signals and erroneous estimation of the physiological parameters. Various attempts have been made to find strategies to circumvent these artifacts, ranging from the use of extra hardware, e.g. accelerometers $[16,4,6,12]$ to several methods of signal processing $[11,8,10]$.

Kim et al. (2007) tried to find a relation between the distorted PPG signal and the acceleration data [6]. They placed the PPG sensor and the accelerometer on the forehead. To have a comparable signal for reference they placed another PPG sensor on the finger (the hand was constrained to avoid all movement). The subject is then asked to shake his head in each of the axes of the accelerometer, thereby finding the corresponding correlation for all axes.

The detected PPG signal is also thought to be additively corrupted by the motion detected with the noise reference (accelerometer) [12]. Nevertheless, cancellation of these movement artifacts by means of the acceleration data is a complex process that requires extensive knowledge of how body acceleration affects the measured PPG signal. For example, knowledge on parameters such as the delay between the two signals, the weight the motion has on the PPG signal, as well as the weight of each accelerometer axis individually, is essential.

During PPG measurement, the influence of the movement condition in a walking/running exercise is particularly strong. Nevertheless, using the 3 -axis accelerometer data recorded together with the PPG measurements, it should be possible to extract a 'clean'
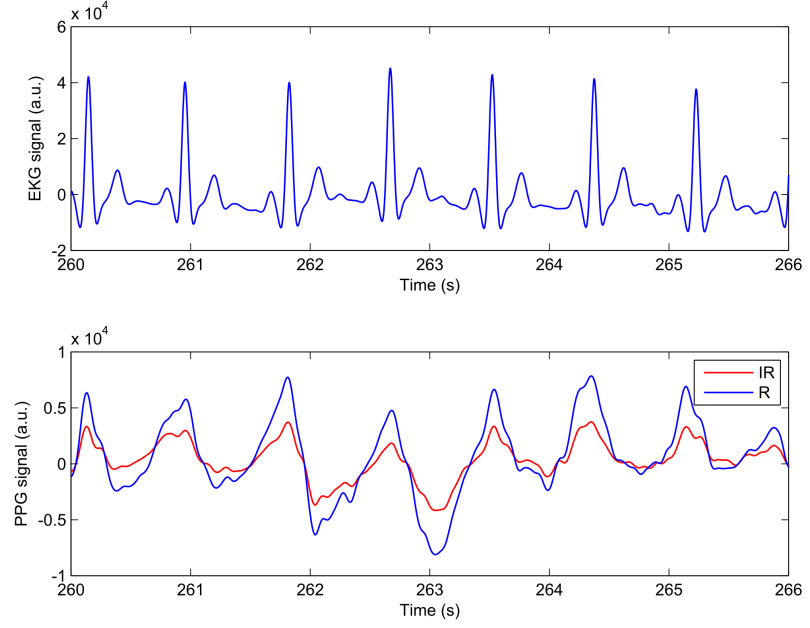

Figure 4: Obtained ECG and PPG signals of a healthy subject walking at $2 \mathrm{~km} / \mathrm{h}$.

PPG from a PPG with artifacts. Studies on reduction of motion artifacts without using extra hardware (e.g. accelerometers) have been performed. However, in those studies only a single motion (e.g. a single movement of a finger) is provoked, allowing to use another part of the body as a PPG reference, or the rest of the signal for adaptive filters. Naraharisetti et al. (2011) compared five signal processing methods to reduce artifacts on a PPG signal, and concluded that the singular value decomposition (SVD) and Cycle-byCycle Fourier Series Analysis provided better results [8]. Other studies used synthetic reference signals, instead of accelerometers, for the adaptive filtering technique $[11,10]$. To our knowledge, no studies on a running person or in a constant motion condition have been performed in the way presented here.

\subsection{Measurement scenario}

The present study was performed indoors using a treadmill. Measurements were recorded of a healthy 28 -year-old male volunteer running continuously at speeds of $0,2,4,9,4,11$ and $0 \mathrm{~km} / \mathrm{h}$ with a duration of about $2 \mathrm{~min}$ each. The measurements were performed together with an ECG (SOMNOlab2, Weinmann Geräte für Medizin GmbH + Co. KG, Hamburg, Germany) used as reference measurement. The device used was a customized in-ear PPG sensor (as described above). In addition, movements were also detected and recorded with an accelerometer. The PPG sensor was fitted in the left ear and the sensor interface was attached to the trunk of the volunteer.

\section{Results and Discussion}

To analyze the pulse waves of a PPG signal, the DC component of the signal has to be removed. Therefore, to get the heart frequency range from the PPG signal, 

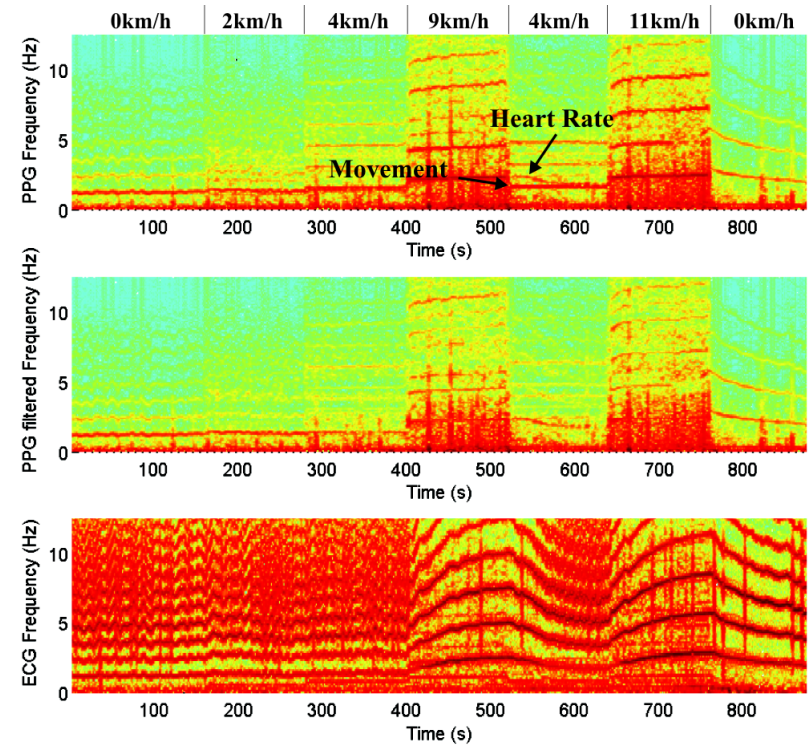

Figure 5: Spectrograms of the PPG, the PPG after removal of motion artifacts, and the reference ECG signals of a healthy subject during the complete measurement.

a 3rd order band-pass Butterworth filter with a cutoff frequency of $0.1 \mathrm{~Hz}$ to $10 \mathrm{~Hz}$ was applied to our signals. To illustrate the performance of the system in a non-active condition, Figure 3 shows a comparison of the reference signal (ECG) and the PPG signal of the subject at $0 \mathrm{~km} / \mathrm{h}$ (standing).

Figure 4 shows a comparison between the reference signal (ECG) and the PPG signal of the subject at $2 \mathrm{~km} / \mathrm{h}$ (walking). Relative to the $0 \mathrm{~km} / \mathrm{h}$ (standing) and $2 \mathrm{~km} / \mathrm{h}$ (walking), no further filter is necessary to correctly detect heart activity once the obtained signal is clear and stable.

Figure 5 presents the time-varying spectrum of the PPG, the filtered PPG and ECG signals. On the ECG spectrogram, the lines are clear and correspond to the subject's heart rate and its harmonics. Note that although the frequencies in the PPG signal measured with our system are not particularly sharp, the behavior is similar to the ECG measured with a commercial device (except at 9 and $11 \mathrm{~km} / \mathrm{h}$ ).

To separate cardiac and motion activity, an intelligent filter has been implemented that uses the accelerometer data ( $y$-axis): the most dominant maxima of the spectral density of the accelerometer data are obtained. By means of these frequencies $f_{\max , \text { accl }}$, boundaries are defined in the following way:

$$
\begin{aligned}
f_{\text {down }} & =f_{\text {max }, \text { accl }}-0.1 \mathrm{~Hz}, & & \text { for main frequencies } \\
f_{\text {up }} & =f_{\text {max }, \text { accl }}+0.1 \mathrm{~Hz}, & & \text { for main frequencies } \\
f_{\text {down }} & =f_{\text {max }, \text { accl }} * n-0.2 \mathrm{~Hz}, & & \text { for } n \text { harmonics } \\
f_{\text {up }} & =f_{\text {max }, \text { accl }} * n+0.2 \mathrm{~Hz}, & & \text { for } n \text { harmonics } \\
\text { with } n & =2,3 \text { and } 4 . & &
\end{aligned}
$$
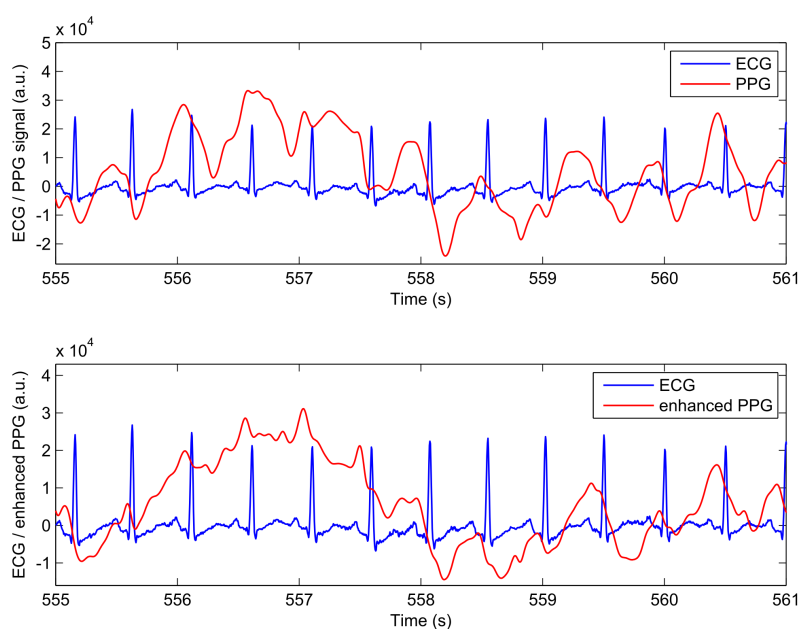

Figure 6: Comparison of the ECG with the normal (upper graph) and the enhanced PPG signals (lower graph), at a speed of $4 \mathrm{~km} / \mathrm{h}$.

These calculated frequencies are used to implement zero-phase digital band-stop filters, which are applied to the sum of both PPG signals (1536th order at main frequency, 512th order at harmonics). The results of this algorithm can be seen in Figure 5 (middle band). When the speed is decreased from 9 to $4 \mathrm{~km} / \mathrm{h}(t=$ $520 \mathrm{~s}$ ), the difference in cardiac and movement activity is clearly seen, since the heart rate slowly decreases whereas the movement remains constant. Thus, the algorithm is able to separate the two activities and significantly reduces motion artifacts; this results in the filtered PPG having a similar spectrum to that derived with the ECG signal. For the recordings at 9 and $11 \mathrm{~km} / \mathrm{h}$, because the main frequencies of the movement artifact are very similar to that of the heart rate, and the amplitude of the provoked artifacts is too high, the present filtering method is not sufficiently reliable. This means that an improved adaptive filter with additional parameters needs to be developed in the future.

To demonstrate the actual enhancement at a speed of $4 \mathrm{~km} / \mathrm{h}$, the resulting signal is shown in Figure 6 . As can be seen, the missing PPG peak at $557.5 \mathrm{~s}$ was recovered in the enhanced PPG and all the other peaks also appear to have a better correlation with the ECG signal. The enhanced PPG shows a better performance in terms of heart activity than the normal PPG signal.

Figure 7 presents data corresponding to the 3 -axis accelerometer obtained at a speed of $9 \mathrm{~km} / \mathrm{h}$. As can be seen the three axes are different, comprised of different waveforms and different amplitudes. A way to correctly reconstruct the PPG signal at higher speeds might be to subtract these data from the PPG [12]. Therefore, this information needs to be carefully analyzed in further studies.

Figure 8 illustrates the spectral densities corre- 

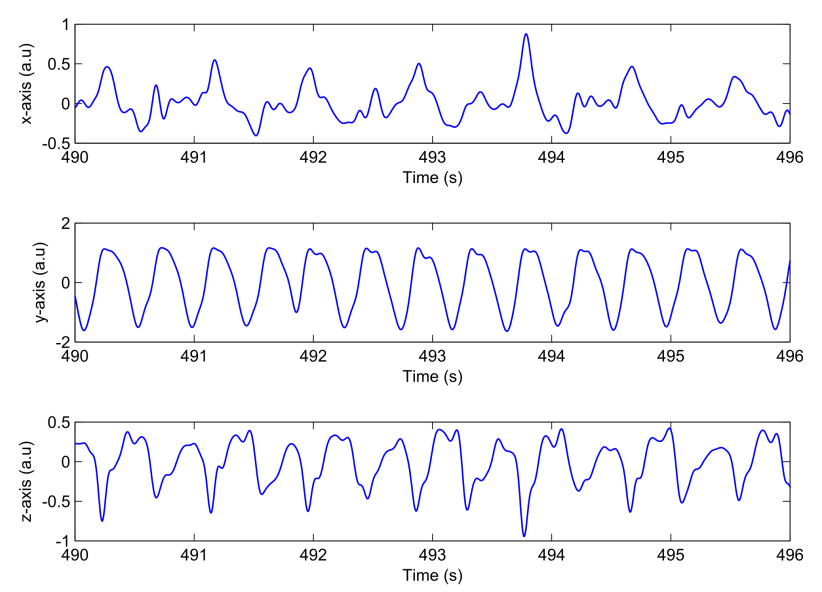

Figure 7: Accelerometer data corresponding to the $x, y$ and $z$-axis, at a speed of $9 \mathrm{~km} / \mathrm{h}$.

sponding to the accelerometer, PPG and ECG of a subject running at a speed of $11 \mathrm{~km} / \mathrm{h}$. It can be seen that the main frequency components measured by the accelerometer that correspond to the movement is very strongly present on the PPG spectrum, overlapping a large part of the heart frequencies, which then become imperceptible.

In this case, reduction of movement artifacts using the same method as used for a speed of $4 \mathrm{~km} / \mathrm{h}$ could not be applied. As can be seen on the ECG spectrum, the heart activity has frequency components in the same range as the movement. This means that if we completely eliminate the movement frequencies the frequencies from heart activity would also be eliminated.

\section{Conclusion}

The PPG-based system proved to be sufficiently robust and stable for speeds up to $2 \mathrm{~km} / \mathrm{h}$ (walking). Without any additional signal processing this system would probably be suitable for daily tasks that do not include extreme movements. For speeds above $4 \mathrm{~km} / \mathrm{h}$, a high order band-stop filter, with cut-off frequencies corresponding to the higher frequency and the next three harmonics of the accelerometer, was implemented. It was then possible to remove the movement artifacts from the PPG signal, thereby revealing the frequency band of heart activity. For higher speeds, such as 9 and $11 \mathrm{~km} / \mathrm{h}$ (running), adaptive filters based on the accelerometer data require additional study in order to get acceptable results.

Our department is also investigating measurement of arterial oxygen saturation $\left(\mathrm{SpO}_{2}\right)$ using the same LAVIMO system. Therefore, it is planned to extend this system with $\mathrm{SpO}_{2}$ measurements during physical activity.
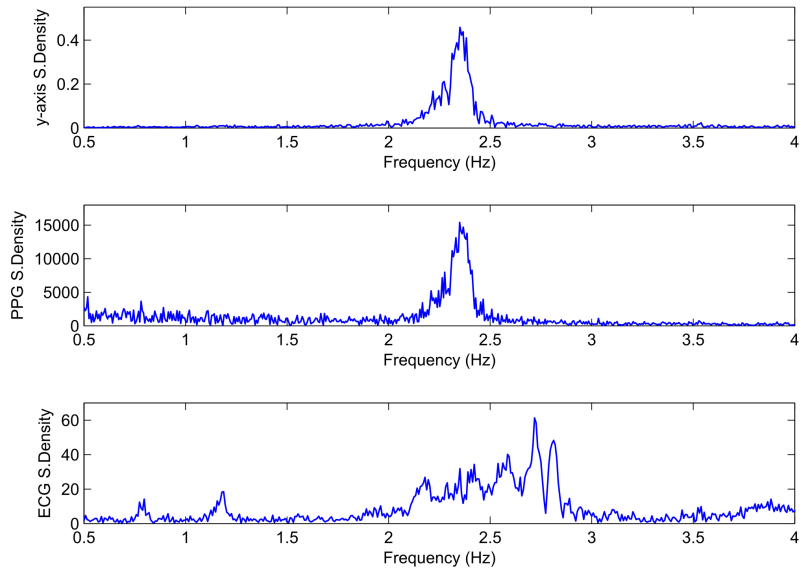

Figure 8: Spectral densities of the accelerometer, PPG and ECG of a healthy subject running at $11 \mathrm{~km} / \mathrm{h}$. Note that the ECG spectrum has a wide range of frequencies because the subject went from a $4 \mathrm{~km} / \mathrm{h}$ to an $11 \mathrm{~km} / \mathrm{h}$ speed exercise (physical stress provoked an increase in heart rate). The PPG is dominated by the movement: the PPG and y-axis density almost correspond with each other.

\section{Acknowledgements}

This research was supervised by Prof. V. Blazek, RWTH Aachen University, Germany.

The authors gratefully acknowledge financial support from the German Federal Ministry of Science and Education, the State of North Rhine-Westphalia (NRW, Germany) and the European Union (NRW Ziel2 Program).

\section{References}

[1] John Allen. Photoplethysmography and its application in clinical physiological measurement. Physiological Measurement 28(3):R1-R39, 2007.

[2] R. Budgett. Fatigue and underperformance in athletes: The overtraining syndrome. British Journal of Sports Medicine 32(2):107-110, 1998.

[3] A. C Burton. The range and variability of the blood flow in the human fingers and the vasomotor regulation of body temperature. American Journal of Physiology - Legacy Content $\mathbf{1 2 7}(3)$ :437-453, 1939.

[4] M. El-Khoury, J. Sola, V. Neuman, J. Krauss. Portable SpO2 monitor: A fast response approach. pp. 1-5. IEEE, 2007.

[5] A.A.R. Kamal, J.B. Harness, G. Irving, A.J. Mearns. Skin photoplethysmography - a review. Computer Methods and Programs in Biomedicine 28(4):257-269, 1989. 
[6] Sang Hyun Kim, Dong Wan Ryoo, Changseok Bae. Adaptive noise cancellation using accelerometers for the PPG signal from forehead. pp. 25642567. IEEE, 2007.

[7] Barry J. Maron. Sudden death in young athletes. New England Journal of Medicine 349(11):10641075, 2003.

[8] Kali Vara Prasad Naraharisetti, Manan Bawa. Comparison of different signal processing methods for reducing artifacts from photoplethysmograph signal. pp. 1-8. IEEE, 2011.

[9] Meir Nitzan, Sergei Turivnenko, Adina Milston, et al. Low-frequency variability in the blood volume and in the blood volume pulse measured by photoplethysmography. Journal of Biomedical Optics 1:223-229, 1996.

[10] M. Raghu Ram, K. Venu Madhav, E. Hari Krishna, et al. A novel approach for motion artifact reduction in PPG signals based on AS-LMS adaptive filter. IEEE Transactions on Instrumentation and Measurement 2011.

[11] M. Raghu Ram, K. Venu Madhav, E. Hari Krishna, et al. On the performance of time varying step-size least mean Squares(TVS-LMS) adaptive filter for MA reduction from PPG signals. pp. 431-435. IEEE, 2011.
[12] A.R. Relente, L.G. Sison. Characterization and adaptive filtering of motion artifacts in pulse oximetry using accelerometers. vol. 2, pp. 1769 1770. IEEE, 2002.

[13] B. Venema, N. Blanik, V. Blazek, et al. Advances in reflective oxygen saturation monitoring with a novel in-ear sensor system: Results of a human hypoxia study. IEEE Transactions on Biomedical Engineering 59(7):2003-2010, 2012.

[14] Boudewijn Venema. Long-term monitoring of cardiac and respiratory activity using optoelectronic sensor. Proceedings of the 15th International Student Conference on Electrical Engineering POSTER 2011 pp. 1-4, 2011.

[15] S. Vogel, M. Hulsbusch, T. Hennig, et al. In-ear vital signs monitoring using a novel microoptic reflective sensor. IEEE Transactions on Information Technology in Biomedicine 13(6):882-889, 2009.

[16] Levi B. Wood, H. Harry Asada. Noise cancellation model validation for reduced motion artifact wearable PPG sensors using MEMS accelerometers. pp. 3525-3528. IEEE, 2006. 